\title{
DEC2 is a negative regulator for the proliferation and differentiation of chondrocyte lineage-committed mesenchymal stem cells
}

\author{
TOMOKO SASAMOTO $^{1 *}$, KATSUMI FUJIMOTO ${ }^{2 *}$, MASAMI KANAWA ${ }^{3}$, JUNKO KIMURA $^{2}$, JUNPEI TAKEUCHI $^{2}$, \\ NAOKO HARADA $^{2}$, NORIKO GOTO ${ }^{4}$, TAKESHI KAWAMOTO ${ }^{2}$, MITSUHIDE NOSHIRO $^{2}$, \\ KETUT SUARDITA $^{2,5}$, KAZUO TANNE ${ }^{1}$ and YUKIO KATO $^{2}$
}

\begin{abstract}
Departments of ${ }^{1}$ Orthodontic Medicine and ${ }^{2}$ Dental and Medical Biochemistry, Institute of Biomedical and Health Sciences, ${ }^{3}$ Natural Science Center for Basic Research and Development, ${ }^{4}$ Department of Pediatric Dentistry, Institute of Biomedical and Health Sciences, Hiroshima University, Hiroshima 734-8553, Japan; ${ }^{5}$ Department of Conservative Dentistry,

Faculty of Dentistry, Airlangga University, Surabaya, East Java 60132, Indonesia
\end{abstract}

Received February 5, 2016; Accepted June 15, 2016

DOI: $10.3892 / \mathrm{ijmm} .2016 .2660$

\begin{abstract}
Differentiated embryo chondrocyte 2 (DEC2) is a basic helix-loop-helix-Orange transcription factor that regulates cell differentiation in various mammalian tissues. DEC2 has been shown to suppress the differentiation of mesenchymal stem cells (MSCs) into myocytes and adipocytes. In the present study, we examined the role of DEC2 in the chondrogenic differentiation of human MSCs. The overexpression of DEC2 exerted minimal effects on the proliferation of MSCs in monolayer cultures with the growth medium under undifferentiating conditions, whereas it suppressed increases in DNA content, glycosaminoglycan content, and the expression of several chondrocyte-related genes, including aggrecan and type $\mathrm{X}$ collagen alpha 1, in MSC pellets in centrifuge tubes under chondrogenic conditions. In the pellets exposed to chondrogenesis induction medium, DEC2 overexpression downregulated the mRNA expression of fibroblast growth factor 18 , which is involved in the proliferation and differentiation of chondrocytes, and upregulated the expression of pl6INK4, which is a cell cycle
\end{abstract}

Correspondence to: Dr Katsumi Fujimoto, Department of Dental and Medical Biochemistry, Institute of Biochemical and Health Sciences, Hiroshima University, 1-2-3 Kasumi, Minami-ku, Hiroshima 734-8553, Japan

E-mail: kfujimo@hiroshima-u.ac.jp

*Contributed equally

Abbreviations: MSCs, mesenchymalstemcells;DEC2,differentiated embryo chondrocyte 2; FGF, fibroblast growth factor; GAG, glycosaminoglycan; $A C A N$, aggrecan; $A L P L$, alkaline phosphatase liver/bone/kidney; COL2A1, type II collagen alpha 1; mCOL10A1, type $\mathrm{X}$ collagen alpha $1 ; O S X$, osterix

Key words: mesenchymal stem cells, differentiated embryo chondrocyte 2, chondrogenesis, proliferation, fibroblast growth factor 18 inhibitor. These findings suggest that DEC2 is a negative regulator of the proliferation and differentiation of chondrocyte lineage-committed mesenchymal cells.

\section{Introduction}

Differentiated embryo chondrocyte 2 (DEC2; also known as SHARP-1 or BHLHE41) is a member of the basic helix-loop-helix-Orange (bHLH-O) family, characterized by a basic DNA binding domain, a helix-loop-helix (HLH) dimerization domain, and Orange extended dimerization domain $(1,2)$. DEC2 is a transcriptional repressor similar to other bHLH-O proteins such as HES and HESR $(3,4)$. The DEC2 homodimer directly binds to the E-box sequence (CACGTG) and represses the transcription of target genes through the recruitment of a corepressor, histone deacetylase $1(3,5)$. DEC2 also antagonizes the transcriptional activity of other transcription factors, including BMAL1 and SREBP-1, by direct protein-protein interactions and/or by competing for binding to the E-box $(5,6)$. Furthermore, DEC2 modulates various biochemical processes, including circadian rhythm (5,7-9), cancer metastasis $(10,11)$, proliferation (12-16) and differentiation $(3,4,17,18)$.

$D E C 2$ is ubiquitously expressed in varying amounts in both embryonic and adult tissues $(1,19)$. The expression level of $D E C 2$ is high in developing limbs, muscle and brain. In our preliminary experiments, $D E C 2$ was expressed at higher levels in mesenchymal stem cells (MSCs) than in fibroblasts (data not shown). MSCs have the capacity to differentiate into a number of types of cells including adipocytes, chondrocytes, osteoblasts and smooth muscle cells (20). Previous studies have shown that DEC2 suppresses myogenesis and adipogenesis of MSCs by inhibiting the transcriptional activities of $\mathrm{MyoD}$ and C/EBP, respectively $(3,4,17)$. These observations suggest that DEC2 may be involved in maintaining MSCs in an undifferentiated state. However, whether DEC2 modulates differentiation of MSCs into osteoblasts or chondrocytes remains unclear.

In preliminary experiments, the overexpression of DEC 2 in human MSCs suppressed chondrogenic differentiation, whereas 
it had a marginal effect on osteogenic differentiation (data not shown). Therefore, we focused on the role of DEC2 in chondrogenic differentiation of MSCs. The results revealed that DEC2 is a negative regulator for the proliferation and differentiation of chondrocyte lineage-committed MSCs, although it has little effect on the proliferation of undifferentiated MSCs. We also examined the effects of DEC2 overexpression on the expressions of fibroblast growth factors (FGFs) and cell cycle-related genes, as these genes play important roles in the proliferation and/or differentiation of chondrogenic cells $(21,22)$.

\section{Materials and methods}

Cells. Human iliac bone marrow MSCs were obtained from RIKEN (Tsukuba, Japan). The cells were maintained in Dulbecco's modified Eagle's medium (DMEM; Sigma, St. Louis, MO, USA) supplemented with $10 \%$ fetal bovine serum (FBS; HyClone, Logan, UT, USA), 1 ng/ml FGF2 (Kaken Pharmaceutical, Tokyo, Japan) and 1\% antibiotic-antimycotic solution (Sigma) at $37^{\circ} \mathrm{C}$ in $5 \% \mathrm{CO}_{2}$. MSCs harvested at the 4th-6th passage were used in the experiments.

Chondrogenic differentiation in pellet cultures. The MSCs were centrifuged at $500 \mathrm{x} \mathrm{g}$ for $5 \mathrm{~min}$ in $15-\mathrm{ml}$ conical polypropylene tubes to form pellets $\left(2.5 \times 10^{5}\right.$ cells/pellet $)$. The pellets were cultured at $37^{\circ} \mathrm{C}$ in a humidified atmosphere of $5 \% \mathrm{CO}_{2}$ for 3 days, with $0.5 \mathrm{ml}$ chondrogenesis induction medium consisting of $\alpha$-minimum essential medium ( $\alpha$-MEM) supplemented with $100 \mathrm{nM}$ dexamethasone, $50 \mu \mathrm{g} / \mathrm{ml}$ ascorbic acid-2-phosphate, $4.5 \mathrm{mg} / \mathrm{ml} \mathrm{D}$-glucose (all from Sigma), $100 \mu \mathrm{g} / \mathrm{ml}$ sodium pyruvate (Wako Pure Chemical, Osaka, Japan), 1\% ITS+ Premix (Thermo Fisher Scientific, Waltham, MA, USA), and $10 \mathrm{ng} / \mathrm{ml}$ TGF- $\beta 3$ (Peprotech, Rocky Hill, NJ, USA). Thereafter, the cells were fed every other day with $1 \mathrm{ml}$ fresh medium.

Low-density monolayer cultures. The MSCs were seeded at a low density $\left(700\right.$ cells $\left./ \mathrm{cm}^{2}\right)$ on 6-well plates for RNA isolation or 96-well plates for the proliferation assay and incubated with DMEM supplemented with $10 \% \mathrm{FBS}$ at $37^{\circ} \mathrm{C}$ in a $\mathrm{CO}_{2}$ incubator.

Construction of an adenoviral expression vector. The recombinant adenovirus containing Dec2 (Ad-Dec2) was constructed using the Adeno-X Expression System (Clontech, Mountain View, CA, USA). Full-length mouse Dec2 (mDec2) cDNA (1) was inserted into the NheI and XbaI sites of the pShuttle vector to generate the expression cassette under the regulation of the cytomegalovirus promoter. The expression cassette was ligated to Adeno-X viral DNA. The adenoviral vector pAd-Dec2 was digested with PacI and then transfected into 293 cells using Lipofectamine (Invitrogen, Carlsbad, CA, USA). The resulting adenoviruses were further propagated in 293 cells and purified using the Adeno- $\mathrm{X}^{\mathrm{TM}}$ purification kit (Clontech). Viral titers were determined using the Adeno- $\mathrm{X}^{\mathrm{TM}}$ rapid titer kit (Clontech). The recombinant adenovirus containing lacZ (Ad-LacZ) was prepared as a negative control (23). MSCs were mock-infected or infected with Ad-Dec2 or Ad-LacZ at the indicated multiplicity of infection (MOI) for $2 \mathrm{~h}$.

Western blot analysis. MSCs were infected with Ad-Dec2 at the indicated MOI (control: MOI =0), and then incubated in DMEM supplemented with $10 \%$ FBS. The cells were lysed with Laemmli buffer, and the lysates were subjected to sodium dodecyl sulfate-polyacrylamide gel electrophoresis (SDS-PAGE). The proteins were transferred to a PVDF membrane (Millipore, Bedford, MA, USA). The membrane was incubated overnight with rabbit antibodies specific for DEC2 $\left(1: 2,000\right.$ dilution at $\left.4^{\circ} \mathrm{C}\right)$. After washes with TBST buffer (25 mM TBS, $0.05 \%$ Tween-20, $\mathrm{pH} 7.4$ ), the membrane was incubated for $1 \mathrm{~h}$ with alkaline phosphatase-conjugated anti-rabbit IgG (1:2,000 dilution at room temperature; Dako, Carpinteria, CA, USA). The antibody-bound bands were visualized using nitro blue tetrazolium and 5-bromo-4chloro-3-indolyl phosphate (Nacalai Tesque, Kyoto, Japan). The anti-DEC2 antibodies were produced by immunizing synthetic peptide fragment Cys-Asn-Pro-Glu-Ser-Ser-GlnGlu-Asp-Ala-Thr-Gln-Pro-Ala. The obtained antibodies were purified by affinity column chromatography.

5-bromo-4-chloro-3-indolyl- $\beta$-D-galactopyranoside (X-gal) staining. MSCs were cultured for 2 days after infection with Ad-LacZ at the indicated MOI. The transduction efficiency of MSCs with the adenovirus was estimated by staining with X-gal (Wako Pure Chemical). The cells were fixed with phosphate-buffered saline (PBS) containing 2\% formaldehyde and $0.2 \%$ glutaraldehyde (both from Wako Pure Chemical) for 5 min, washed twice with PBS and incubated in X-gal staining solution overnight at $37^{\circ} \mathrm{C}$. After rinsing in water, images of the cells were captured using an Olympus IX70 microscope and an Olympus DP20 camera (Olympus, Tokyo, Japan).

Proliferation in monolayer culture. Following mock infection or infection with Ad-Dec2 or Ad-LacZ at the indicated MOI, MSCs were seeded at a low density $\left(700\right.$ cells $\left./ \mathrm{cm}^{2}\right)$ on 96 -well plates, and incubated with DMEM supplemented with $10 \% \mathrm{FBS}$ at $37^{\circ} \mathrm{C}$ in a $\mathrm{CO}_{2}$ incubator for 4 days. Cell proliferation was estimated with a Cell Counting kit-8 (CCK-8; Dojindo, Kumamoto, Japan) using WST- 8 according to the manufacturer's instructions.

Reverse transcription-quantitative PCR (RT-qPCR). Chondrocyte pellets were frozen in liquid nitrogen and crushed using an SK-Mill (Tokken, Chiba, Japan). Total RNA was prepared using an RNeasy kit (Qiagen, Hilden, Germany) and reverse transcribed with ReverTra Ace (Toyobo, Osaka, Japan) according to the manufacturer's instructions. The synthesized First-Strand cDNA was used for PCR amplification with specific primers and TaqMan probe sets (Table I). Quantitative PCR analyses were performed using an ABI PRISM 7900HT Sequence Detection System instrument and software (Applied Biosystems Inc., Foster City, CA, USA) based on the $\Delta \Delta \mathrm{Cq}$ method (24). Data were normalized against 18S rRNA levels. The PCR cycling conditions included an incubation of $50^{\circ} \mathrm{C}$ for $2 \mathrm{~min}$ and a denaturation of $95^{\circ} \mathrm{C}$ for $10 \mathrm{~min}$, which was followed by 40 cycles of $95^{\circ} \mathrm{C}$ for $15 \mathrm{sec}$ and $60^{\circ} \mathrm{C}$ for $1 \mathrm{~min}(25)$.

Toluidine blue staining. In order to estimate the extent of proteoglycan accumulation in the pellets, the diameter of the pellets was measured using a ruler, and toluidine blue staining was performed. The pellets were fixed in $10 \%$ buffered formalin, and embedded in paraffin. Sections of $5 \mathrm{~mm}$ were prepared and stained with toluidine blue (26). The sections 
Table I. Primer and probe sequences used for RT-qPCR.

\begin{tabular}{|c|c|c|}
\hline Gene & Primer $\left(5^{\prime} \rightarrow 3^{\prime}\right)$ & Probe $\left(5^{\prime} \rightarrow 3^{\prime}\right)$ \\
\hline$A C A N$ & $\begin{array}{l}\text { F: AGTATCATCAGTCCCAGAATCTAGCA } \\
\text { R: GGAATGCAGAGGTGGTTTCAC }\end{array}$ & AGACGTCCGCCTATCCTGAAGCTG \\
\hline$A L P L$ & $\begin{array}{l}\text { F: AGACGTCCGCCTATCCTGAAGCTG } \\
\text { R: GCCATACAGGATGGCAGTGA }\end{array}$ & CCCCATGCTGAGTGACACAGACAAGAA \\
\hline COL2A1 & $\begin{array}{l}\text { F: GAGACAGCATGACGCCGAG } \\
\text { R: GGCTGCGGATGCTCTCAAT }\end{array}$ & ATGCCACACTCAAGTCCCTCAACAACCA \\
\hline COL10A1 & $\begin{array}{l}\text { F: CTAGTATCCTTGAACTTGGTTCATGGA } \\
\text { R: ACTGTGTCTTGGTGTTGGGTAGTG }\end{array}$ & CGCTGAACGATACCAAACGCCCAC \\
\hline OSX & $\begin{array}{l}\text { F: ATGAGCTGGAGCGTCATGTG } \\
\text { R: AGGTGGTCGCTTCGGGTAA }\end{array}$ & TCACCTGCCTGCTCTGCTCCAAGC \\
\hline$h D E C 2$ & $\begin{array}{l}\text { F: GCATCAGAAGATAATTGCTTTACAGAA } \\
\text { R: TCTCAAACCGGGAGAGGTATTG }\end{array}$ & CGTTCCACTCGGGATTTCAAACATGC \\
\hline$m D e c 2$ & $\begin{array}{l}\text { F: ATTGCTTTACAGAATGGGGAGCG } \\
\text { R: AAAGCGCGCGAGGTATTGCAAGAC }\end{array}$ & CGACTTGGATGCGTTCCACTCGG \\
\hline$F G F 2$ & $\begin{array}{l}\text { F: TTCTTCCTGCGCATCCAC } \\
\text { R: TGCTTGAAGTTGTAGCTTGATGT }\end{array}$ & Roche Universal Probe \#7 \\
\hline$F G F 7$ & $\begin{array}{l}\text { F: GCAAAGTAAAAGGGACCCAAG } \\
\text { R: TCACTTTCCACCCCTTTGAT }\end{array}$ & Roche Universal Probe \#59 \\
\hline FGF 18 & $\begin{array}{l}\text { F: ATGAACCGCAAAGGCAAG } \\
\text { R: GAACACACACTCCTTGCTGGT }\end{array}$ & Roche Universal Probe \#60 \\
\hline Cyclin D1 & $\begin{array}{l}\text { F: GAAGATCGTCGCCACCTG } \\
\text { R: GACCTCCTCCTCGCACTTCT }\end{array}$ & Roche Universal Probe \#67 \\
\hline$p 21$ & $\begin{array}{l}\text { F: TCACTGTCTTGTACCCTTGTGC } \\
\text { R: GGCGTTTGGAGTGGTAGAAA }\end{array}$ & Roche Universal Probe \#32 \\
\hline pl6INK4 & $\begin{array}{l}\text { F: GTGGACCTGGCTGAGGAG } \\
\text { R: CTTTCAATCGGGGATGTCTG }\end{array}$ & Roche Universal Probe \#34 \\
\hline
\end{tabular}

$A C A N$, aggrecan; $A L P L$, alkaline phosphatase liver/bone/kidney; COL2A1, type II collagen alpha 1; COL1OA1, type X collagen alpha 1; OSX, osterix; $h D E C 2$, human differentiated embryo chondrocyte $2 ; m D e c 2$, mouse differentiated embryo chondrocyte $2 ; F G F$, fibroblast growth factor; F, forward; R, reverse.

were then examined with an Olympus IX70 microscope and an Olympus DP20 camera.

Quantification of glycosaminoglycan $(G A G)$ and DNA content. The pellets were washed twice with phosphate-buffered saline (PBS) and digested with $0.3 \mathrm{mg} / \mathrm{ml}$ papain (Wako Pure Chemical) in $50 \mathrm{mM}$ phosphate buffer, $\mathrm{pH} 6.5$, containing $2 \mathrm{mM}$ EDTA and $2 \mathrm{mM} \mathrm{N}$-acetyl-cysteine (Wako Pure Chemical) at $60^{\circ} \mathrm{C}$. The papain-digested extracts were assayed for GAG and DNA content. Sulfated GAG content was quantified using the Blyscan ${ }^{\mathrm{TM}}$ Sulfated Glycosaminoglycan assay kit (Biocolor, Newtownabbey, UK) according to the manufacturer's instructions. The DNA content of the pellets was determined using the Quant-iT ${ }^{\mathrm{TM}}$ PicoGreen dsDNA assay kit (Thermo Fisher Scientific) with lambda DNA as a standard.

Statistical analysis. All experiments were performed at least in triplicate. Data are expressed as the means \pm standard devia- tion (SD). One-way ANOVA followed by Dunnett's post hoc test was performed to determine the significance of differences in multiple comparisons. $\mathrm{P}<0.05$ was considered to indicate a statistically significant difference.

\section{Results}

Endogenous expression of DEC2 during chondrogenic differentiation of MSCs. To examine the role of DEC2 in chondrocyte differentiation, we examined the changes in the expression levels of $D E C 2$ and the main chondrocyte marker genes, namely aggrecan $(A C A N)$, alkaline phosphatase liver/bone/kidney (ALPL), type II collagen alpha 1 (COL2A1), type X collagen alpha $1(C O L 10 A 1)$ and osterix $(O S X)$ in MSC pellet cultures after exposure to chondrogenesis induction medium (Fig. 1). The mRNA levels of COL2A1, OSX and $A C A N$ started to increase on day 7, and then those of $A L P L$ and COL1OAl started to increase on day 14 . The expression 

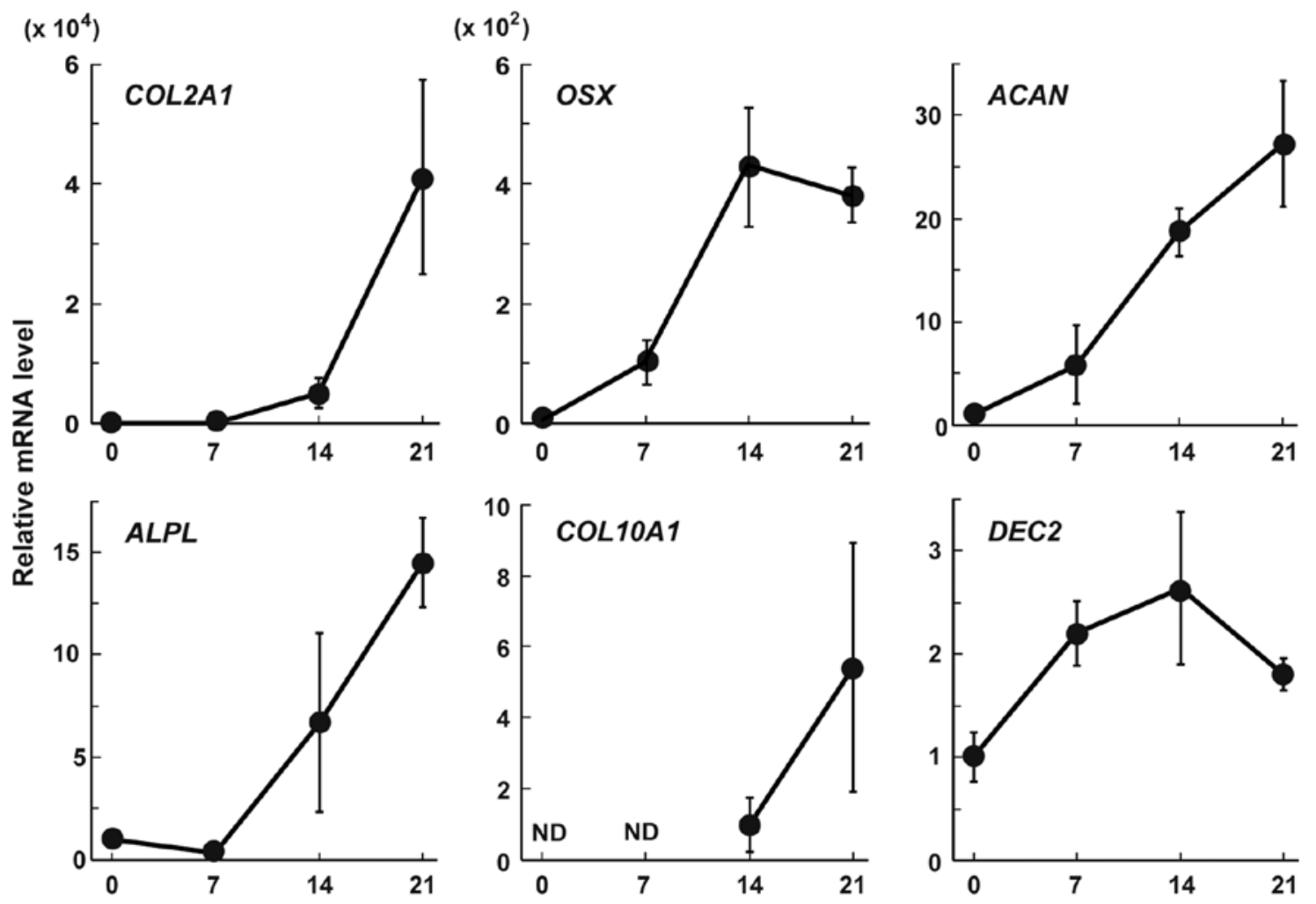

Days after chondrogenic induction

Figure 1. Time course of gene expression of chondrocyte marker genes and differentiated embryo chondrocyte 2 (DEC2) during chondrogenic differentiation of mesenchymal stem cells (MSCs). MSCs in pellet cultures were exposed to chondrogenesis induction medium. On the indicated days after the induction of differentiation, total RNA was extracted from the MSC pellets, and the mRNA levels of chondrocyte marker genes (COL2A1, OSX, ACAN, ALPL and COL1OA1) and DEC2 were determined by RT-qPCR. Data represent the means \pm SD of fold-changes relative to expression of each mRNA on day 0 , except for COL1OA1 ( $\mathrm{n}=3)$. The expression level of COL10A1 mRNA is relative to that on day 14 because no mRNA was detected on days 0 and 7. ND, not detected.

levels of these mRNAs, with the exception of that of $O S X$, continued to increase at least until day 21. DEC2 mRNA expression started to increase on day 7 and then decreased on day 21 .

Effect of DEC2 overexpression on MSC proliferation in monolayer cultures. MSC monolayer cultures were infected with Ad-Dec2. The DEC2 protein levels successfully increased in an MOI-dependent manner up to an MOI of 500 in MSCs infected with Ad-Dec2 (Fig. 2A), and a high level of DEC2 expression in MSCs was maintained until at least 7 days after infection with Ad-Dec2 (data not shown). In similar cultures, the transduction efficiency of the adenovirus was determined with Ad-LacZ. It increased the intensity of $\beta$-galactosidase staining in an MOI-dependent manner, and $>90 \%$ of the cells showed positive staining for $\beta$-galactosidase at an MOI of 500 (Fig. 2B), suggesting that this method allows the overexpression of DEC2 in most MSCs.

To examine the effect of DEC2 overexpression on cell proliferation, MSCs were infected with either Ad-Dec2 or Ad-LacZ at various MOI levels and incubated at a low density with DMEM supplemented with $10 \%$ FBS. On day 4, there was no significant difference with regard to proliferation between Ad-Dec2- and Ad-LacZ-infected cells, and infection with Ad-Dec2 or Ad-LacZ did not show cytotoxicity up to an MOI of 500 (Fig. 2C).
Effects of DEC2 overexpression on extracellular matrix formation during chondrogenesis. MSCs were infected with either Ad-Dec2 or Ad-LacZ at an MOI of 500 and then exposed to chondrogenesis induction medium in pellet cultures. The size of these pellets gradually increased after the onset of differentiation (Fig. 3A). However, pellets of Ad-Dec2-infected cells were smaller than those of Ad-LacZ- or mock-infected cells. Further, the diameter of Ad-Dec2-infected cell pellets was significantly smaller than that of Ad-LacZ- or mock-infected cell pellets on day 28 (Fig. 3B). There were no differences between the diameters of Ad-LacZ- and mock-infected cell pellets.

To estimate the extent of proteoglycan accumulation in the pellets, we performed toluidine blue staining. On days 21 and 28, proteoglycan deposition (characterized by a red-purple color) was observed at high levels in the Ad-LacZ- and mock-infected pellets; however, it was scarcely detected in the Ad-Dec2-infected pellets (Fig. 3C). Microscopic analysis also indicated the accumulation of abundant proteoglycans and typical lacunae structures around chondrocytes in both the Ad-LacZ- and mock-infected pellets on days 21 and 28, whereas the proteoglycan accumulation and lacunae structures were scarcely observed in the Ad-Dec2-infected pellets.

We subsequently quantified the total GAG content per pellet (Fig. 4A), along with the GAG content per microgram DNA on day 21 (Fig. 4B and C). The total GAG content and GAG/DNA of the Ad-Dec2-infected pellets were lower than that 

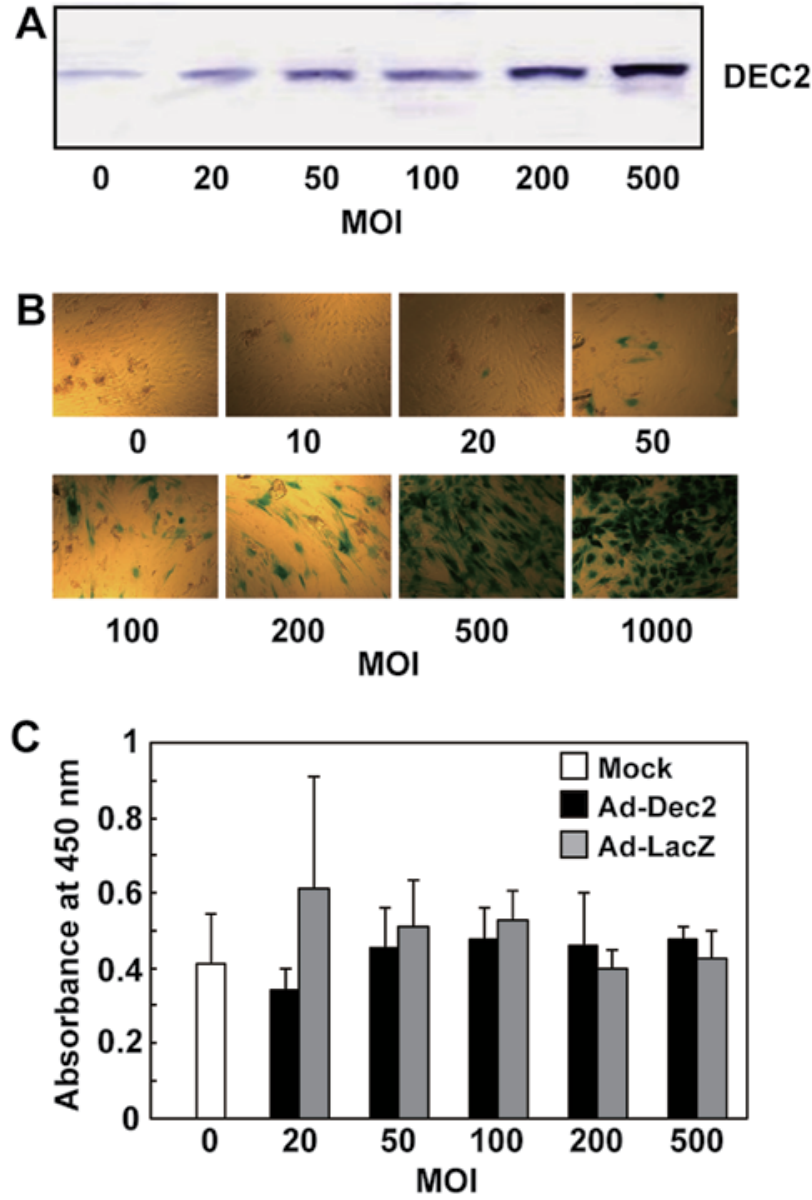

Figure 2. Effect of differentiated embryo chondrocyte 2 (DEC2) overexpression on the proliferation of mesenchymal stem cells (MSCs) in monolayer culture (A) Confluent monolayers of MSCs were infected with Ad-Dec2 at the indicated multiplicity of infection (MOI) and the expression levels of DEC2 were determined by western blot analysis using anti-DEC2 antibody. (B) Confluent monolayers of MSCs were infected with adenovirus containing lacZ (Ad-LacZ) at the indicated MOI and the expression levels of LacZ were determined by staining with X-gal (magnification, x200). (C) MSCs were mock-treated or infected with either Ad-Dec2 or Ad-LacZ at the indicated MOI, and then seeded at a low density $\left(700\right.$ cells $\left./ \mathrm{cm}^{2}\right)$ on 96 -well plates. After 4 days of culture, cell proliferation was measured using the WST- 8 assay. Data represent the means $\pm \mathrm{SD}(\mathrm{n}=3)$.

in the Ad-LacZ- and mock-infected pellets (Fig. 4A and C). In addition, a significant decrease in the DNA content per pellet was observed in the Ad-Dec2-infected pellets in comparison with the Ad-LacZ-infected pellets, indicating the suppression of cell growth by DEC2 (Fig. 4B). By contrast, there were no significant differences between the Ad-LacZ- and mock-infected pellets with respect to the GAG content, DNA content and GAG/DNA.

Effects of DEC2 overexpression on the expression of chondrocyte-related genes. We examined the effects of DEC2 overexpression on the expression of chondrocyte-related genes in the pellet cultures. DEC2 overexpression suppressed the expression of $A C A N$ and COL1OAl, but not the expression of $O S X$ and $A L P L$, on day 28 (Fig. 5A). The inhibitory effect of DEC2 on ACAN and COL1OA1 expression was not observed on day 7 , suggesting that DEC2 may indirectly suppress the expression of the two chondrocyte genes. We also examined the expression levels of Dec2 in DEC2-overexpressing MSC pellets. The $m D e c 2$ mRNA level was $\sim 80$-fold higher than the endogenous human expression on day 7 , and the high-level expression of $m D e c 2$ decreased to less than one tenth on day 28 (Fig. 5A), suggesting that the infection with Ad-Dec2 affects the chondrogenic potential of MSCs in the early stage.

Because FGFs play an important role in the control of proliferation and differentiation of chondrocytes $(21,22,27)$, we also examined whether DEC2 affected the expression of some FGFs. DEC2 overexpression strongly suppressed the expression of $F G F 18$, but not that of $F G F 2$ and $F G F 7$, on days 1 and 7 (Fig. 5B).

Effects of DEC2 overexpression on the expression of cell cycle-related genes. The regulation of cell cycle progression is closely associated with cell proliferation and differentiation (28). Therefore, we examined whether DEC2 overexpression in MSCs altered the expression levels of the cell cycle-related genes, namely cyclin D1,p21 and p16INK4. Cyclin D1 acts as a positive regulator of cell cycle progression, whereas p21 and p16INK4 negatively regulate the cell cycle by inhibiting cyclin-dependent kinases (CDKs) (28). The mRNA expression of p16INK4 was upregulated on day 1 by DEC 2 overexpression in the MSC pellets exposed to chondrogenesis induction medium but not in the monolayer cultures exposed to growth medium (Fig. 5C and D). In the pellets, DEC2 overexpression also increased the average level of p21 and decreased cyclin D1 expression levels, although the observed differences were not statistically significant (Fig. 5C). These findings suggest that DEC2 suppresses cell cycle progression of the mesenchymal cells in pellet cultures exposed to the differentiation induction medium by regulating the expression of cell cycle-related genes in a stage-dependent manner.

\section{Discussion}

In this study, we demonstrated that DEC2 acts as a negative regulator of chondrogenic differentiation. The forced expression of DEC2 resulted in the inhibition of both cell proliferation and GAG accumulation during chondrogenesis in MSC pellet cultures, whereas in repeated experiments, it did not suppress the proliferation of MSC monolayer cultures. These findings indicate that DEC2 inhibits the proliferation of chondrocyte lineage-committed MSCs, but not undifferentiated MSCs. In pellet cultures of MSCs, the expression of $D E C 2$ began to increase earlier than that of chondrocyte markers, such as $A L P L$ and COL10A1, and then decreased in the late differentiation stage, suggesting that DEC2 inhibits chondrogenic differentiation of MSCs in premature stage, and may delay the maturation of chondrocytes.

DEC2 suppresses the proliferation of various types of cells, including human lung cancer cell lines (A549, NCI-H520 and NCI-H596 cells), human epidermoid carcinoma cells (HEp3), human mammary epithelial cells (HMECs), and NIH3T3 cells (12-15), although DEC2 has little effect on the proliferation of HepG2 cells (13). DEC2-induced cell growth arrest is reportedly associated with decreased cyclin $\mathrm{Dl}$ expression in $\mathrm{C} 2 \mathrm{C} 12$ and NCI-H520 cells as well as HMECs $(4,13,14)$. Consistent with these findings, the DEC2-mediated inhibition of proliferation in MSC pellet cultures was associated with a decrease in the average cyclin Dl levels and an increase in the average p16INK4 and $p 21$ levels. However, DEC2 exerted 

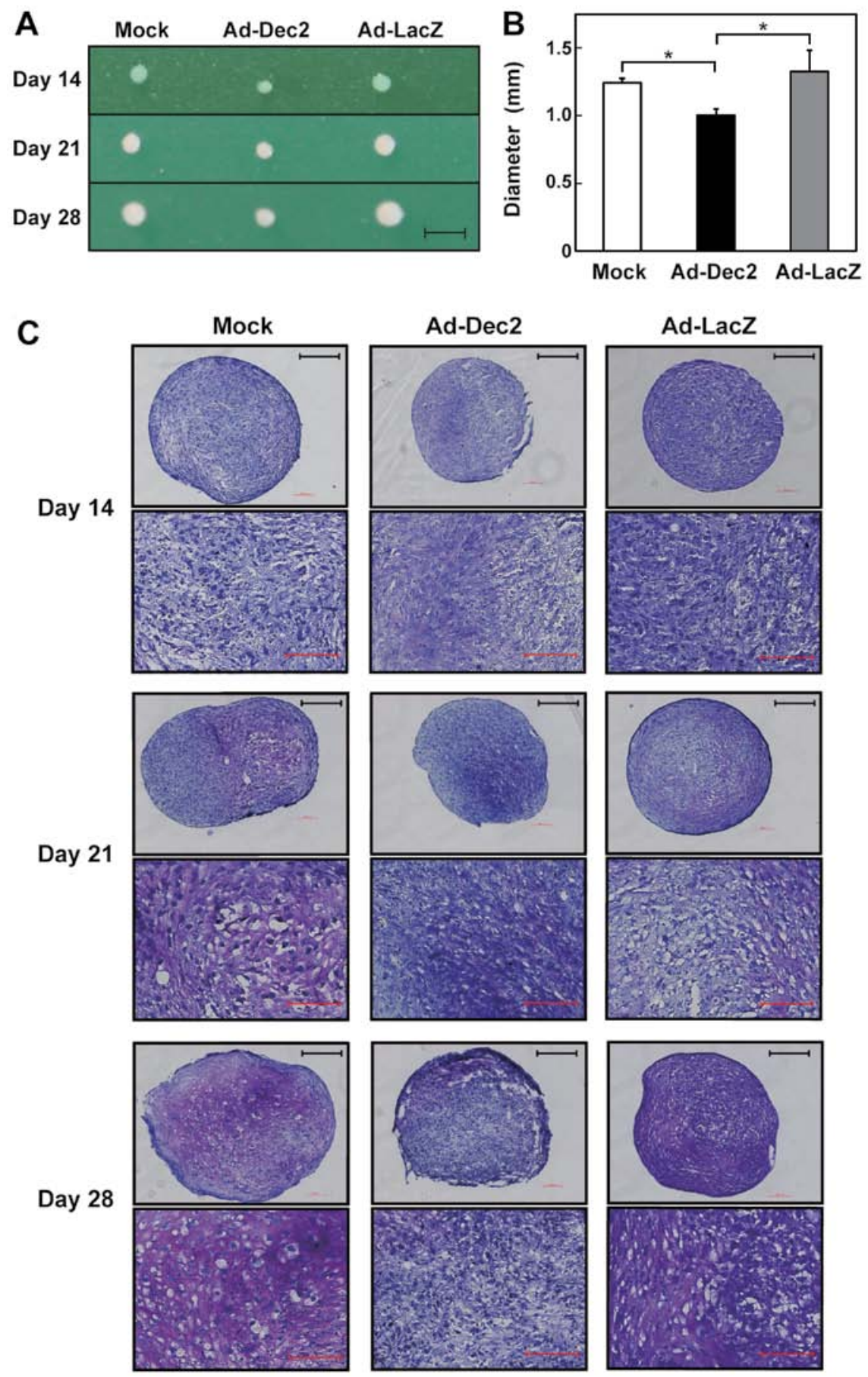

Figure 3. Morphological and histological analysis of chondrogenic pellet cultures of mesenchymal stem cells (MSCs) overexpressing differentiated embryo chondrocyte 2 (DEC2). Following mock infection or infection with Ad-Dec2 [multiplicity of infection (MOI) of 500] or adenovirus containing lacZ (Ad-LacZ) (MOI of 500), MSCs were induced to differentiate into chondrocytes. The extent of chondrogenic differentiation was evaluated by the following morphological and histological analysis. (A) Gross observation of MSC pellets on days 14, 21 and 28 after chondrogenic induction. Scale bars, $2 \mathrm{~mm}$. (B) Size measurement (diameter) of MSC pellets on day 28 after chondrogenic induction. Data represent the means $\pm \mathrm{SD}(\mathrm{n}=3)$. $\mathrm{P}<0.05$. (C) Toluidine blue staining of MSC pellets on days 14, 21 and 28 after chondrogenic induction. Overview (upper panels) and magnified (lower panels) images of the pellets are shown. Upper black scale bars, $200 \mu \mathrm{m}$; lower red scale bars, $100 \mu \mathrm{m}$. The results presented are typical of three independent experiments.

minimal effects on MSC proliferation, and the expression of pl6INK4 in the undifferentiated state in low-density monolayer cultures. Taken together, these findings suggest that the growth inhibitory effect of DEC2 depends upon cell type, cell density, and/or differentiation stage. However, we have not examined the effect of DEC2 on the proliferation and differentiation of other tissue-derived MSCs, including adipose tissue-derived MSCs and umbilical cord-derived MSCs as bone marrow-derived MSCs have been most widely used in studies of chondrogenesis. This issue warrants further investigation in the future using the other tissues-derived MSCs.

FGF signaling has been implicated in the regulation of endochondral bone growth $(21,22)$. Even though FGF3 receptor (FGF3R) activation suppresses the terminal differentiation of growth plate chondrocytes, FGF18, a ligand of FGF3R, may exert a positive effect on the proliferation and 

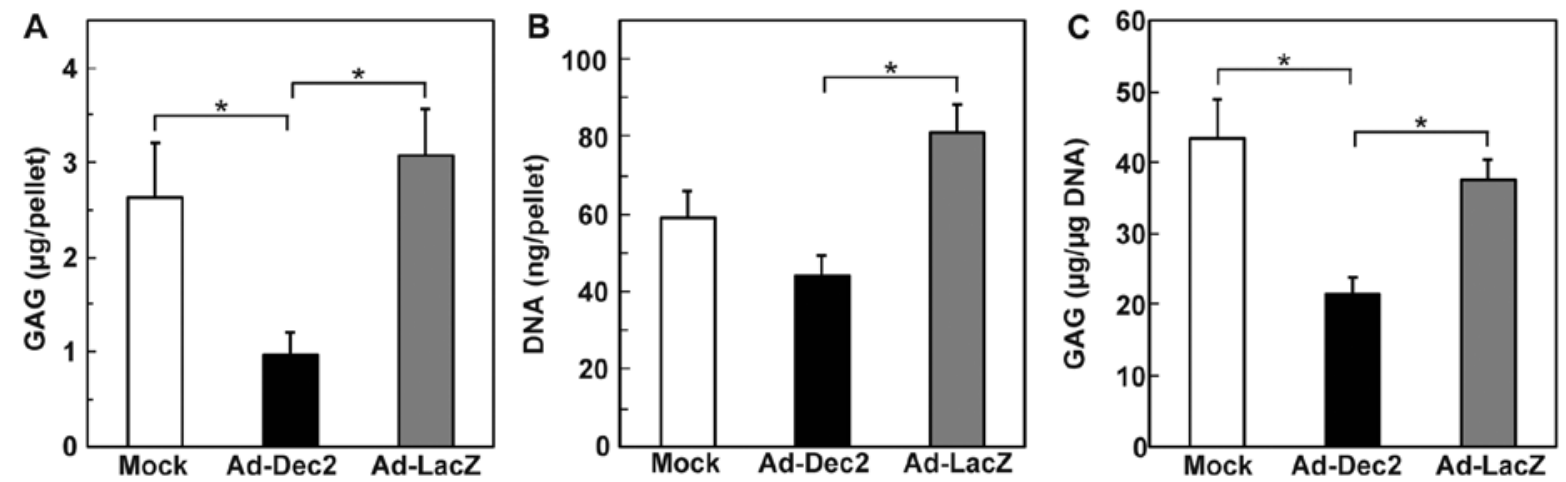

Figure 4. Inhibition of glycosaminoglycan (GAG) synthesis and proliferation of mesenchymal stem cells (MSCs) by the overexpression of differentiated embryo chondrocyte 2 (DEC2). Following mock infection or infection with Ad-Dec2 [multiplicity of infection (MOI) of 500] or adenovirus containing lacZ (Ad-LacZ) (MOI of 500), MSCs were induced to differentiate into chondrocytes. Pellets cultured for 21 days were analyzed for (A) GAG content per pellet, (B) DNA content per pellet and (C) GAG content per microgram of DNA. Data represent the means $\pm \mathrm{SD}(\mathrm{n}=3)$. ${ }^{*} \mathrm{P}<0.05$.
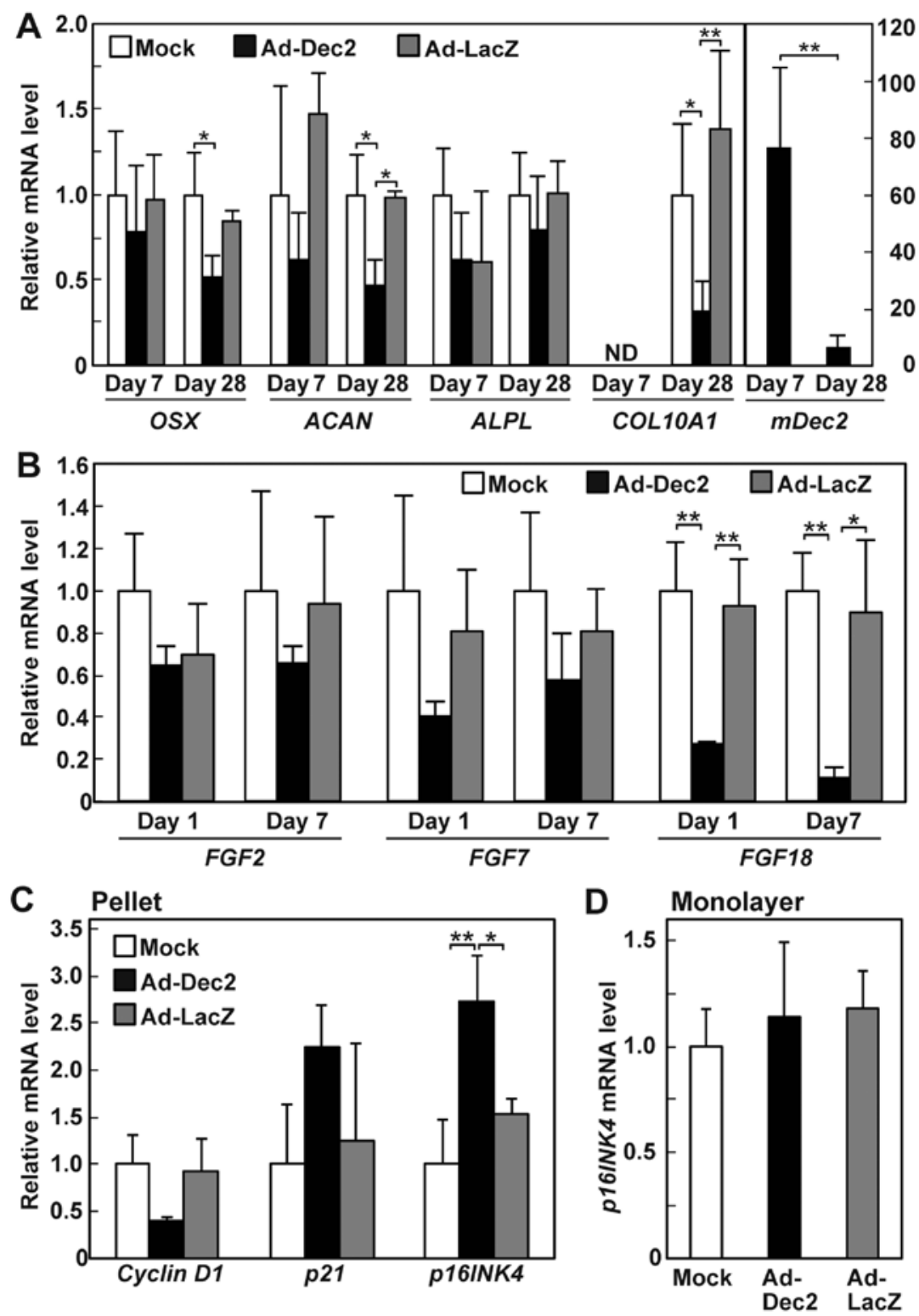

Figure 5. Effects of overexpression of differentiated embryo chondrocyte 2 (DEC2) on the expression of chondrocyte marker genes and cell cycle-related genes in mesenchymal stem cells (MSCs). Following mock infection or infection with Ad-Dec2 [multiplicity of infection (MOI) of 500] or adenovirus containing lacZ (Ad-LacZ) (MOI of 500), MSCs were (A-C) maintained in pellet or (D) monolayer cultures. (B-D) On days 1, (A and B) day 7 and (A) day 28 , total RNA was extracted from MSCs, and the mRNA levels of (A) chondrocyte marker genes and mouse Dec2 (mDec2), (B) fibroblast growth factors (FGFs), (C) cell cycle-related genes and (D) p16INK4 were determined by RT-qPCR. Data represent the means \pm SD fold-changes relative to expression in mock-infected cells, except for $\mathrm{mDec} 2(\mathrm{n}=3)$. The mRNA expression of $\mathrm{mDec} 2$ is relative to that of endogenous human $D E C 2$ on day 7 . ND, not detected. ${ }^{*} \mathrm{P}<0.05$ and ${ }^{* *} \mathrm{P}<0.01$. 
differentiation of immature committed chondrocytes (29-32). $F G F 18$ knockout $\left(F G F 18^{-/}\right)$mice exhibited decreased chondrocyte proliferation activity and a delay in the initiation of chondrocyte hypertrophy at the embryonic stage (29). We found that DEC2 overexpression strongly represses the expression of FGF18. Although the role of FGF18 in cartilage development remains controversial, the signaling may be associated with DEC2-mediated suppression of proliferation and/or differentiation of chondrocyte-lineage committed MSCs.

FGF18 is also involved in cartilage angiogenesis through the upregulation of vascular endothelial grow th factor (VEGF) (29). By contrast, DEC2 represses the expression of Vegf, resulting in the reciprocal expression of Dec2 and Vegf in mouse rib cartilage (33). Therefore, DEC2 and FGF18 appear to exert opposite effects on vasculogenesis as well as chondrogenesis.

DEC1 and DEC2 are structurally similar, but they often have different functions. For example, DEC1 has pro-apoptotic effects, whereas DEC2 has anti-apoptotic effects on TNF- $\alpha$-induced apoptosis in MCF-7 cells (34). Furthermore, DEC2, but not DEC1, suppresses the expression of Vegf through binding with HIF1 $\alpha$ in NIH3T3 cells (33). Previous findings have shown that the overexpression of $\mathrm{DEC1}$ promotes chondrogenic differentiation of rabbit MSCs and mouse ATDC5 cells (23). Therefore, DEC1 and DEC2 seem to have opposite actions on chondrogenesis. Besides direct DNA binding, DEC1 and DEC2 modulate gene expression through protein-protein interactions with common and distinct transcription factors $(3,5,6,8,10,17,33)$. Identifying the partners of DEC2 will clarify how DEC2 regulates the chondrogenic differentiation of MSCs.

In conclusion, we have shown that DEC2 is involved in the suppression of proliferation and differentiation of chondrocyte lineage-committed MSCs. DEC2 inhibits the proliferation of MSCs by regulating the expression of cell cycle-related genes under chondrogenic conditions. The decreased proliferation of MSCs may decrease their subsequent differentiation into chondrocytes. An alternative possibility is that DEC2 suppresses chondrogenic differentiation by downregulating FGF18 independently of MSC proliferation. Besides inhibition of chondrocyte differentiation, DEC2 suppresses the differentiation of MSCs into adipocytes $(17)$ and myoblasts $(3,4)$, suggesting that DEC2 may be implicated in maintaining MSCs in the undifferentiated state.

\section{Acknowledgements}

The present study was supported by Grants-in-Aid for Challenging Exploratory Research (no. 24659876) and Scientific Research (C) (no. 22592067) from the Ministry of Education, Culture, Sports, Science and Technology of Japan. We would like to thank Dr Eiso Hiyama at the Natural Science Center for Basic Research and Development, Hiroshima University for the use of their facilities.

\section{References}

1. Fujimoto K, Shen M, Noshiro M, Matsubara K, Shingu S, Honda K, Yoshida E, Suardita K, Matsuda Y and Kato Y: Molecular cloning and characterization of DEC2, a new member of basic helix-loop-helix proteins. Biochem Biophys Res Commun 280: 164-171, 2001.
2. Sun H, Ghaffari S and Taneja R: bHLH-Orange Transcription Factors in Development and Cancer. Transl Oncogenomics 2: 107-120, 2007.

3. Fujimoto K, Hamaguchi H, Hashiba T, Nakamura $T$, Kawamoto T, Sato F, Noshiro M, Bhawal UK, Suardita K and Kato Y: Transcriptional repression by the basic helix-loop-helix protein Dec2: Multiple mechanisms through E-box elements. Int J Mol Med 19: 925-932, 2007.

4. Azmi S, Ozog A and Taneja R: Sharp-1/DEC2 inhibits skeletal muscle differentiation through repression of myogenic transcription factors. J Biol Chem 279: 52643-52652, 2004.

5. Honma S, Kawamoto T, Takagi Y, Fujimoto K, Sato F, Noshiro M, Kato Y and Honma K: Dec1 and Dec2 are regulators of the mammalian molecular clock. Nature 419: 841-844, 2002.

6. Choi SM, Cho HJ, Cho H, Kim KH, Kim JB and Park H: Stra13/DEC1 and DEC2 inhibit sterol regulatory element binding protein-1c in a hypoxia-inducible factor-dependent mechanism. Nucleic Acids Res 36: 6372-6385, 2008

7. Hamaguchi H,Fujimoto K, Kawamoto T, Noshiro M, Maemura K, Takeda N, Nagai R, Furukawa M, Honma S, Honma K, et al: Expression of the gene for Dec2, a basic helix-loop-helix transcription factor, is regulated by a molecular clock system. Biochem J 382: 43-50, 2004.

8. Kato Y, Kawamoto T, Fujimoto $\mathrm{K}$ and Noshiro $\mathrm{M}$ : DEC1/STRA13/SHARP2 and DEC2/SHARP1 coordinate physiological processes, including circadian rhythms in response to environmental stimuli. Curr Top Dev Biol 110: 339-372, 2014.

9. Rossner MJ, Oster H, Wichert SP, Reinecke L, Wehr MC, Reinecke J, Eichele G, Taneja R and Nave KA: Disturbed clockwork resetting in Sharp-1 and Sharp-2 single and double mutant mice. PLoS One 3: e2762, 2008.

10. Montagner M, Enzo E, Forcato M, Zanconato F, Parenti A, Rampazzo E, Basso G,Leo G, Rosato A, Bicciato S, et al: SHARP1 suppresses breast cancer metastasis by promoting degradation of hypoxia-inducible factors. Nature 487: 380-384, 2012.

11. Sato F, Kawamura H, Wu Y, Sato H, Jin D, Bhawal UK, Kawamoto T, Fujimoto K, Noshiro M, Seino H, et al: The basic helix-loop-helix transcription factor DEC2 inhibits TGF- $\beta$ induced tumor progression in human pancreatic cancer BxPC-3 cells. Int J Mol Med 30: 495-501, 2012.

12. Adam AP, George A, Schewe D, Bragado P, Iglesias BV, Ranganathan AC, Kourtidis A, Conklin DS and Aguirre-Ghiso JA: Computational identification of a p38SAPK-regulated transcription factor network required for tumor cell quiescence. Cancer Res 69: 5664-5672, 2009.

13. Falvella FS, Colombo F, Spinola M, Campiglio M, Pastorino U and Dragani TA: BHLHB3: a candidate tumor suppressor in lung cancer. Oncogene 27: 3761-3764, 2008.

14. Li Y, Shen Q, Kim HT, Bissonnette RP, Lamph WW, Yan B and Brown PH: The rexinoid bexarotene represses cyclin D1 transcription by inducing the DEC2 transcriptional repressor. Breast Cancer Res Treat 128: 667-677, 2011.

15. Liu JJ, Chung TK, Li J and Taneja R: Sharp-1 modulates the cellular response to DNA damage. FEBS Lett 584: 619-624, 2010.

16. Wu Y, Sato H, Suzuki T, Yoshizawa T, Morohashi S, Seino H, Kawamoto T, Fujimoto K, Kato Y and Kijima H: Involvement of c-Myc in the proliferation of MCF-7 human breast cancer cells induced by bHLH transcription factor DEC2. Int J Mol Med 35: 815-820, 2015.

17. Gulbagci NT, Li L, Ling B, Gopinadhan S, Walsh M, Rossner M, Nave KA and Taneja R: SHARP1/DEC2 inhibits adipogenic differentiation by regulating the activity of C/EBP. EMBO Rep 10: 79-86, 2009.

18. Yang XO, Angkasekwinai P, Zhu J, Peng J, Liu Z, Nurieva R, Liu X, Chung Y, Chang SH, Sun B and Dong C: Requirement for the basic helix-loop-helix transcription factor Dec2 in initial TH2 lineage commitment. Nat Immunol 10: 1260-1266, 2009.

19. Azmi S and Taneja R:Embryonic expression of mSharp-1/mDEC2, which encodes a basic helix-loop-helix transcription factor. Mech Dev 114: 181-185, 2002.

20. Pittenger MF, Mackay AM, Beck SC, Jaiswal RK, Douglas R, Mosca JD, Moorman MA, Simonetti DW, Craig S and Marshak DR: Multilineage potential of adult human mesenchymal stem cells. Science 284: 143-147, 1999.

21. Horton WA and Degnin CR: FGFs in endochondral skeletal development. Trends Endocrinol Metab 20: 341-348, 2009.

22. Ellman MB, Yan D, Ahmadinia K, Chen D, An HS and Im HJ: Fibroblast growth factor control of cartilage homeostasis. J Cell Biochem 114: 735-742, 2013. 
23. Shen M, Yoshida E, Yan W, Kawamoto T, Suardita K, Koyano Y, Fujimoto K, Noshiro M and Kato Y: Basic helix-loop-helix protein DEC1 promotes chondrocyte differentiation at the early and terminal stages. J Biol Chem 277: 50112-50120, 2002.

24. Livak KJ and Schmittgen TD: Analysis of relative gene expression data using real-time quantitative PCR and the 2(-Delta DeltaC(T)) method. Methods 25: 402-408, 2001

25. Fujii S, Fujimoto K, Goto N, Kanawa M, Kawamoto T, Pan H, Srivatanakul P, Rakdang W, Pornprasitwech J, Saskianti T, et al: Characteristic expression of, and in dental pulp cells. Biomed Rep 3: 566-572, 2015.

26. Suardita K, Fujimoto K, Oda R, Shimazu A, Miyazaki K, Kawamoto T and Kato Y: Effects of overexpression of membranebound transferrin-like protein (MTf) on chondrogenic differentiation in Vitro. J Biol Chem 277: 48579-48586, 2002.

27. Correa D, Somoza RA, Lin P, Greenberg S, Rom E, Duesler L, Welter JF, Yayon A and Caplan AI: Sequential exposure to fibroblast growth factors (FGF) 2, 9 and 18 enhances hMSC chondrogenic differentiation. Osteoarthritis and cartilage/OARS. Osteoarthritis Res Soc 23: 443-453, 2015.

28. Budirahardja Y and Gönczy P: Coupling the cell cycle to development. Development 136: 2861-2872, 2009.

29. Liu Z, Lavine KJ, Hung IH and Ornitz DM: FGF18 is required for early chondrocyte proliferation, hypertrophy and vascular invasion of the growth plate. Dev Biol 302: 80-91, 2007.
30. Liu Z, Xu J, Colvin JS and Ornitz DM: Coordination of chondrogenesis and osteogenesis by fibroblast growth factor 18 . Genes Dev 16: 859-869, 2002.

31. Yamaoka H, Nishizawa S, Asawa Y, Fujihara Y, Ogasawara T, Yamaoka K, Nagata S, Takato T and Hoshi K: Involvement of fibroblast growth factor 18 in dedifferentiation of cultured human chondrocytes. Cell Prolif 43: 67-76, 2010.

32. Mori Y, Saito T, Chang SH, Kobayashi H, Ladel CH, Guehring H, Chung UI and Kawaguchi H: Identification of fibroblast growth factor-18 as a molecule to protect adult articular cartilage by gene expression profiling. J Biol Chem 289: 10192-10200, 2014.

33. Sato F, Bhawal UK, Kawamoto T, Fujimoto K, Imaizumi T, Imanaka T, Kondo J, Koyanagi S, Noshiro M, Yoshida H, et al: Basic-helix-loop-helix (bHLH) transcription factor DEC2 negatively regulates vascular endothelial growth factor expression. Genes Cells 13: 131-144, 2008.

34. Wu Y, Sato F, Bhawal UK, Kawamoto T, Fujimoto K, Noshiro M, Morohashi S, Kato Y and Kijima H: Basic helix-loop-helix transcription factors DEC1 and DEC2 regulate the paclitaxel-induced apoptotic pathway of MCF-7 human breast cancer cells. Int J Mol Med 27: 491-495, 2011. 